\title{
Efectividad de una intervención de enfermería sobre abordaje al tabaquismo en personas con un trastorno mental
}

Effectiveness of a Nursing Intervention on Approaching Smoking Cessation in People with a Mental Disorder

\author{
María Rosario Valera Fernández ${ }^{1-3}$, Ana María González Cuello², Rosa Aceña Domínguez ${ }^{1}$, \\ Ana Belén Valera Fernández ${ }^{1}$, Antonio Rafael Moreno Poyato ${ }^{3}$, Francina Fonseca Casals ${ }^{1}$
}

${ }^{1}$ Departamento de Adicciones. Instituto de Neuropsiquiatría i Adicciones (INAD). Consorci Mar Parc de Salut. Barcelona, España. ${ }^{2}$ Departamento de Enfermería. Escuela Universitaria de Enfermería de la Universidad de Murcia. Murcia, España.

${ }^{3}$ Departamento de Enfermería. Escuela Superior de Enfermería del Mar, adscrita a la Universidad Pompeu Fabra. Barcelona, España. Contacto: charo-valera@hotmail.com

Fecha de recepción: 15 de mayo de 2019 / Fecha de aceptación: 16 de noviembre de 2019

\begin{abstract}
Resumen
Introducción: Se ha encontrado una importante relación entre el consumo de tabaco y distintos trastornos relacionados con la salud mental, observándose una mayor prevalencia de consumo, mayor dependencia a la nicotina, menores intentos de abandono y mayor dificultad para conseguir la abstinencia, respecto a la población general.

Objetivo: Evaluar la efectividad de una intervención de enfermería sobre abordaje al tabaquismo para generar motivación en la reducción del consumo de tabaco o una cesación tabáquica completa a personas con un trastorno mental grave.

Metodología: Estudio cuasiexperimental pre-post, en el que participaron 25 usuarios de los servicios de hospitalización parcial, completa y atención comunitaria. Se realiza una entrevista inicial y una intervención grupal.

Resultados: Se reduce el número de cigarrillos en 9.4 cigarrillos/día y se aumenta el grado de autoeficacia. Se mantiene el grado de dependencia a la nicotina y la motivación para dejar de fumar. Mejora de los valores de cooximetría y tensión arterial.

Conclusiones: Las personas con un trastorno mental están motivadas e interesadas en dejar su consumo de tabaco.
\end{abstract}

Palabras clave: tabaco, trastorno mental, intervención, enfermería, hospitalización.

\begin{abstract}
Introduction: An important relationship has been found between tobacco use and various mental health-related disorders, with a higher prevalence of consumption, greater dependence on nicotine, lower attempts at abandonment and greater difficulty in achieving withdrawal, with respect to general population

Objective: To evaluate the effectiveness of a nursing intervention on smoking approach to generate motivation in the reduction of tobacco consumption or a complete smoking cessation to people with a serious mental disorder.

Methodology: Pre-post quasi-experimental study, in which 25 users of partial, complete hospitalization and community care services participated. An initial interview and a group intervention is carried out.

Results: The number of cigarettes is reduced by 9.4 cigarettes/day and the degree of self-efficacy is increased. The degree of nicotine dependence and the motivation to quit smoking are maintained. Improvement of blood pressure and blood pressure values.

Conclusions: People with a mental disorder are motivated and interested in quitting their tobacco use.
\end{abstract}




\section{Introducción}

¿Por qué casi todos los jugadores (ludópatas) fuman? (...)

¿Por qué todas las prostitutas y todos los locos fuman?

Leo Tolstói (1828-1910)

"Why do men stupefy themselves?"

Original en Essay and Letters (1911)

Aunque ya hace más de 100 años Tolstoi se preguntaba por qué todos los locos fumaban, es en 1986 cuando la comunidad científica empieza a corroborar una fuerte asociación entre el consumo de tabaco y la presencia de sintomatología psiquiátrica ${ }^{1}$. En estos años se ha encontrado una importante relación entre el consumo de tabaco y distintos trastornos relacionados con la salud mental, especialmente depresión, esquizofrenia, trastornos por uso de sustancias y ansiedad ${ }^{2,3}$.

Existe una prevalencia de consumo de tabaco de dos a cinco veces superior entre las personas con un trastorno mental que en la población general ${ }^{4,5}$, o según algunas publicaciones recientes, incluso cinco veces superior $^{6}$. Se estima que en EE. UU. el $70 \%$ de los cigarrillos consumidos por los estadounidenses son fumados por la población con trastornos mentales?

La figura 1 muestra la proporción del consumo de tabaco en función de los diagnósticos psiquiátricos presentes ${ }^{4}$ :

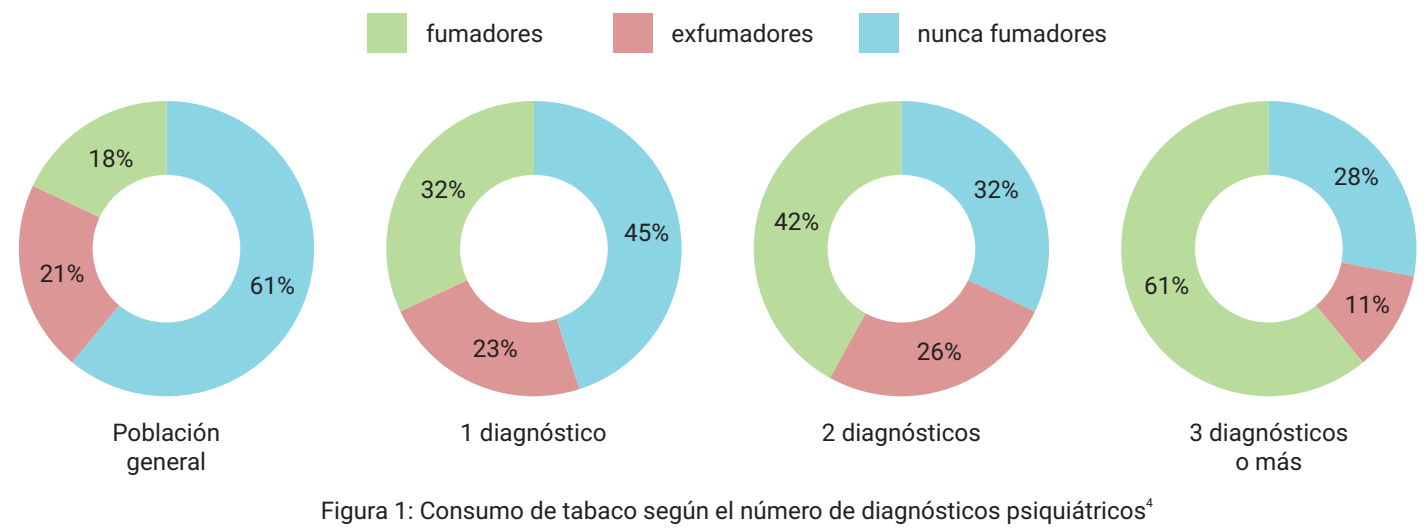

En la población con trastornos mentales también se ha descrito $^{8,9}$ :

- una adicción a la nicotina más elevada, existiendo además una relación directamente proporcional entre la intensidad de la clínica psiquiátrica y la gravedad de adicción;

- un vínculo con el cigarrillo con características propias;

- menor número de intentos de abandono;

- mayor dificultad en la obtención de la abstinencia estable.

Las mayores tasas de prevalencia de consumo tabáquico en personas con un trastorno mental se encuentran dentro del ámbito hospitalario. León y Díaz ${ }^{10} 1 l e-$ varon a cabo un metaanálisis de 42 estudios donde encontraron que la prevalencia de consumo tabáquico era del $68 \%$ en usuarios con esquizofrenia hospitaliza- dos frente al $57 \%$ de los que eran atendidos en el ámbito comunitario.

Son varias las teorías que tratan de explicar la mayor vulnerabilidad al consumo de tabaco en las personas con trastornos mentales. Al igual que en la población general, no existe un único factor que haga más vulnerables a estas personas ${ }^{11}$, siendo el resultado de la combinación de factores biológicos, psicológicos y sociales.

Sobre la cesación tabáquica en la población con trastorno mental, el estudio de Schroeder y Morris ${ }^{12}$ muestra el porcentaje de personas que ha conseguido abandonar el hábito según el tipo de patología psiquiátrica que presenta o la ausencia de la misma. No obstante, ningún trastorno mental es incapacitante para dejar de fumar. Sin embargo, no se llega a los niveles de cesación de la población sin trastornos, encontrán- 
dose grandes diferencias en el caso del trastorno bipo$\operatorname{lar}(16 \%$ frente al $42.5 \%$ de la población no psiquiátrica) y en las personas con trastornos psicóticos $(27.2 \%$ versus $42.5 \%$ ). Los resultados del estudio de Veiga ${ }^{11}$ muestran un fenómeno similar al anterior estudio, observándose cómo las tasas de abstinencia de las personas con un trastorno mental son inferiores a las de la población general.

Este consumo no es abordado por los profesionales de la salud mental con la misma intensidad que por quienes cuidan a personas con otras enfermedades crónicas y recidivantes. El Consejo Internacional de Enfermería (CIE) alienta a este personal sanitario a "integrar la prevención y cesación del consumo de tabaco como parte de su práctica regular de enfermería"13.

El objetivo principal del estudio es evaluar la efectividad de una intervención de enfermería sobre abordaje al tabaquismo en personas con un trastorno mental grave.

\section{Metodología \\ Diseño de estudio}

El estudio siguió una metodología científica basándose en una investigación cuantitativa de tipo analítico cuasiexperimental pre-post.

\section{Población diana}

Se diseñó una intervención enfermera del estudio destinada a usuarios vinculados a los servicios de rehabilitación psiquiátrica de media estancia y alta dependencia psiquiátrica como unidades de hospitalización completa, el hospital de día como servicio de hospitalización parcial y los centros de salud como servicios de atención comunitaria, de la Red de Salud Mental del Institut de Neuropsiquiatria i Addiccions (INAD) del Consorci Mar Parc de Salut de Barcelona. Este estudio obtuvo la aprobación del Comité de Ética del Parc de Salut Mar(2016/6855/I).

\section{Criterios de selección}

Los criterios de inclusión en el estudio eran usuarios/as mayores de edad, de cualquiera de los servicios mencionados anteriormente, que fueran fumadores/as y que presentasen una estabilidad psicopatológica entendida como la ausencia de cambio de tratamiento durante el último mes. La elección de los participantes se realizó de forma voluntaria.

\section{Selección de la muestra}

El tamaño muestral se calculó en base a las diferencias esperadas pre-post respecto a la puntuación obtenida en el test de motivación para dejar de fumar de Richmond. Para un test $\mathrm{t}$ de Student de datos relacionados, con una potencia estadística del $80 \%$ y un error alfa de 0.05 , se necesitaban 24 participantes para detectar como estadísticamente significativa una diferencia equivalente a dos tercios desviación estándar. Se asumió una pérdida máxima de seguimiento del $25 \%$.

\section{Procedimiento}

La intervención sobre abordaje al tabaquismo consta de dos partes:

1. Entrevista inicial. En esta se realizó la recogida de datos iniciales mediante una anamnesis completa sobre datos sociodemográficos y clínicos, incluyendo el control de las medidas biológicas (TA y CO expirado), así como los datos relacionados con el consumo de tabaco como:

a. la dependencia a la nicotina mediante el Test de Fagerström: la versión original consta de 8 ítems, es un instrumento autoaplicado y fue validado en español por Becoña en 1998;

b. la dependencia psicológica, social y gestual del tabaco a través de la Escala de Glover-Nilson: la versión recomendada presenta 11 ítems que valoran los elementos psicológicos, gestuales y sociales implicados en la dependencia. El marco de referencia temporal es el momento actual. Se trata de un instrumento autoaplicado y validado por Nerín en 2005 ;

c. la motivación para dejar de fumar con el Test de Richmond: permite situar a los usuarios en los estadios de cambio de Prochaska y DiClemente, hecho que permite determinar el tipo de intervención a llevar a cabo en cada individuo. Consta de 4 ítems, es un instrumento autoaplicado y fue validado por Córdoba en el año 2000;

d. la autoeficacia del usuario/a ante el abandono tabáquico, el estadio de cambio a través de la University of Rhode Island Change Assessment Scale (URICA): es un autoinforme diseñado para la evaluación de los estadios de cambio según la perspectiva del modelo transteórico de Prochaska y DiCle- 
mente. Está compuesta por 32 ítems, es un instrumento autoaplicado y fue validada en español por Gómez-Peña en 2011;

e. el craving a tabaco mediante el Questionnaire on Smoking Urges-Brief (QSU): la versión original consta de 32 ítems pero la versión utilizada en este caso es la versión simplificada de 10 ítems, evaluados con una escala tipo Likert. Es un instrumento autoaplicado y validado por Cepeda Benito en 2004;

f. el síndrome de abstinencia con la Minnesota Nicotine Withdrawal Scale (MNWS): se trata de un instrumento autoaplicado que consta de 9 ítems y fue validado por Toll en 2007.

2. Intervención grupal. Se empleó una metodología presencial y una visión participativa, dinámica y motivacional. Se realizó una intervención grupal en cada servicio incluido en el estudio. Cada intervención grupal estaba compuesta por 10 sesiones semanales, con una duración de 90 minutos aproximadamente (60 minutos para la intervención psicoeducativa, y 30 minutos adicionales para el control de las medidas biológicas). La intervención era de tipo cíclica, a la cual se podía incorporar un participante en una sesión avanzada sin necesidad de seguir un orden establecido.

El material informativo de las sesiones fue de elaboración propia al no encontrar en la literatura científica material específico para esta población. Este material se elaboró con la información de guías validadas, como el Programa de información sobre tabaquismo de la Región de Murcia del año 2014, la Guía para dejar de fumar del Ministerio de Sanidad, Servicios Sociales e Igualdad y Comité Nacional para la Prevención del Tabaquismo del $2005^{14}$, la guía práctica Recintes sense fum de la Xarxa Catalana d'Hospitals sense fum del $2011^{15}$, la Guía de actuación de pacientes fumadores ingresados en unidades de salud mental de la Xarxa Catalana d'Hospitals sense fum del $2012^{16}$ y la guía clínica práctica Treating Tobacco Use and Dependence: 2008 Update $^{17}$.

La temática trabajada en cada sesión fue:

1. Conocimientos previos. Mitos sobre el tabaco.

2. Conocimientos básicos sobre tabaquismo. Autorregistros.

3. Tipos de cigarrillos. Calendario.

4. Consecuencias del tabaquismo y beneficios de su abandono.

5. Factores motivacionales de mantenimiento y abandono.

6. Síndrome de abstinencia.
7. Tratamiento para la reducción o la cesación completa.

8. Caída y recaída. Utilización de TIC en el tabaquismo.

9. Cigarrillo electrónico. Role-playing.

10. Conocimientos posteriores. Diploma.

\section{Análisis de datos}

Para el análisis estadístico inicial, se describieron las variables cuantitativas mediante la media aritmética y su correspondiente desviación estándar. Las variables categóricas se describieron mediante tabla de frecuencias (número y porcentaje). Se evaluaron posibles asociaciones entre variables categóricas mediante test chi-cuadrado de Pearson (o prueba exacta de Fisher en función de la adecuación de la prueba). Se evaluaron posibles diferencias entre períodos de estudio (pre y post) el test de los rangos con signo de Wilcoxon (muestras pareadas) para las variables continuas. Se consideraron asociaciones estadísticamente significativas aquellas cuyo p-valor era $<0.05$. Los análisis estadísticos se realizaron mediante el paquete estadístico STATA 15.0 software (Stata Corp.; College Station, Texas, USA).

\section{Resultados}

La muestra estuvo formada por 25 participantes, de los cuales el $40 \%(n=10)$ estaban vinculados al servicio de hospitalización completa, el $24 \%(n=6)$ a hospitalización parcial y el $36 \%$ restante $(n=9)$ a atención comunitaria. De éstos, el $56 \%$ eran hombres $(n=14)$ y el $44 \%$ mujeres $(n=9)$, con una edad media de 47.5 años (comprendida entre los 19 y 62 años) $( \pm 7,9)$.

Sobre los diagnósticos, el $48 \%$ de los participantes $(n=12)$ estaban diagnosticados de esquizofrenia, el $16 \%(n=4)$ de trastorno esquizoafectivo, otro $16 \%$ $(n=4)$ de trastorno depresivo, el $12 \%(n=3)$ de trastorno afectivo bipolar y el $8 \%$ restante $(n=2)$ otros diagnósticos. En este caso, se objetivó una diferencia en la distribución de los diagnósticos en función del servicio al que están vinculados, mostrando una mayor proporción de los trastornos psicóticos en los servicios de hospitalización completa y atención comunitaria, frente a la mayor proporción de trastornos afectivos en la hospitalización parcial.

Sobre las características de consumo de los y las participantes, se halló un consumo medio de 27.6 cigarrillos/día durante una media de 30.1 años. Habían 
intentado dejar de fumar en 1.7 ocasiones, objetivándose el doble de intentos de abandono entre los participantes de atención comunitaria frente a los servicios de hospitalización parcial y completa. De los participantes, el $68 \%(\mathrm{n}=17)$ habían presentado un periodo de abstinencia anterior, de los cuales el $80 \%$ referían no haber presentado de forma subjetiva un empeoramiento de la sintomatología psiquiátrica de su trastorno de base durante este periodo de abstinencia. El grado de dependencia psicológica era elevado (11.9), la depen- dencia social moderada (4.1) y el grado de dependencia gestual leve (11.1). Los resultados de la escala QSU mostraron que el nivel de craving (37.4) era elevado, sobre todo en los participantes de los servicios de hospitalización completa y atención comunitaria. Los síntomas abstinenciales valorados mediante la escala MNWS presentaban una puntuación similar en los tres servicios, objetivándose una ligera diferencia no estadísticamente significativa en el servicio de hospitalización parcial (Tabla 1).

\begin{tabular}{|c|c|c|c|c|}
\hline Variables & $\begin{array}{c}\text { Rehabilitación } \\
\text { media y larga } \\
\text { estancia }(n=10)\end{array}$ & $\begin{array}{l}\text { Hos pitalización } \\
\text { parcial } \\
(n=6)\end{array}$ & $\begin{array}{c}\text { Atención } \\
\text { comunitaria } \\
(n=9)\end{array}$ & p \\
\hline \multirow[t]{2}{*}{$\mathrm{N}^{0}$ cigarrillos/día } & $19.3(13.3)$ & $22(9.90)$ & $40.5(5.83)$ & .0008 \\
\hline & & & & .0099 \\
\hline Craving (QSU) & $39.3(20.14)$ & $27.67(10.89)$ & $42(17.04)$ & .3604 \\
\hline Abs tinencia (MNWS) & $11.7(9.22)$ & $9.33(11.34)$ & $11.56(8.26)$ & .6434 \\
\hline \multicolumn{5}{|l|}{ Dependencias } \\
\hline Física (Fagers tröm) & $4.1(2.46)$ & $6.83(1.72)$ & $8.33(1.00)$ & .0303 y .0001 \\
\hline Psicológica (GN) & $11.7(4.62)$ & $11.16(3.92)$ & $12.6(3.16)$ & .7266 \\
\hline Social (GN) & $4.1(1.72)$ & $3.16(2.31)$ & $4.8(1.53)$ & .0416 \\
\hline Gestual (GN) & $9.8(3.48)$ & $9.5(7.23)$ & $13.7(6.28)$ & .2853 \\
\hline Motivación & $3(2.74)$ & $5.5(2.88)$ & $6.67(2.96)$ & .0071 \\
\hline Autoeficacia & $2.7(2.54)$ & $4.17(3.31)$ & $5.67(2.65)$ & .0145 \\
\hline
\end{tabular}

Tabla 1: Comparación de resultados relacionados con el consumo entre los servicios

Tras la intervención, se encontró que respecto al número de cigarrillos consumidos de forma diaria hubo una disminución de 9.4 cigarrillos, resultando estadísticamente significativo (27.6 versus 18.16 ; $\mathrm{p}<0.001)$. El Test de Fagerström, que evalúa la dependencia a la nicotina, descendió en 0.92 puntos, aunque la diferencia no era significativa (6.28 vs 5.36; p: $0.085)$, al igual que con la motivación para dejar de fumar (4.92 vs 5.60; p: 0.202). El grado de autoeficacia y el nivel de conocimientos sobre el tabaquismo también mostraron diferencias significativas antes $\mathrm{y}$ después de la intervención (Tabla 2).

\begin{tabular}{|lccccccc|}
\hline & \multicolumn{2}{c}{ Pre-Intervención } & \multicolumn{2}{c}{ Post-Intervención } & \multicolumn{2}{c|}{ Diferencia } \\
Variable & Media (M) & DE & Media (M) & DE & Media (M) & DE & p \\
\hline N $^{\circ}$ Cigarrillos/día & 27.6 & 14.09 & 18.16 & 12.09 & -9.4 & 10.81 & $\mathbf{. 0 0 0 1}$ \\
Fagerström & 6.28 & 2.62 & 5.36 & 2.72 & -.92 & 2.56 & .1094 \\
Richmond & 4.92 & 3.20 & 5.60 & 2.40 & .68 & 2.59 & .2632 \\
Autoeficacia & 4.12 & 2.96 & 5.32 & 2.23 & 1.20 & 2.08 & $\mathbf{. 0 4 9 0}$ \\
\hline
\end{tabular}

Tabla 2: Diferencias entre variables antes y después de la intervención grupal 
Al analizar los datos relativos a las medidas biológicas controladas, se obtuvieron diferencias estadísticamente significativas en cuanto a la cooximetría $(\mathrm{p}=0.005)$, siendo ésta de 2.28 en el inicio y de 1.68 tras la intervención $(0=$ entre 0 y 6 partes por millón $(\mathrm{ppm}) ; 1=$ entre 7 y 10 ppm; $2=$ entre 11 y 15 ppm; $3=$ entre 16 y 25 ppm; 4 = más de 26 ppm). La tensión arterial disminuyó una media de $2.1 \mathrm{mmHg}$ no resultando estadísticamente significativa $(p=0.080)$. Se objetivó que las cifras de tensión arterial sistólica que estaban dentro de la normalidad (no superando los 140 $\mathrm{mmHg}$ ) se mantenían estables, sin embargo, las cifras arteriales sistólicas por encima de la máxima se veían reducidas.

\section{Discusión}

Los resultados de este estudio sugieren que la intervención propuesta es eficaz para conseguir una disminución del consumo de cigarrillos entre la población con un trastorno mental. De los mismos, se muestran que varias de las percepciones existentes entre el tabaquismo y la salud mental se ponen en duda. Por ejemplo, existe la percepción de que las personas con un trastorno mental no tienen motivación e interés en dejar de fumar $^{18}$. Sin embargo, la motivación encontrada en este estudio fue de un grado moderado. Igualmente existe la creencia de que el hecho de dejar de fumar empeora la evolución de la patología mental y/o adictiva de base en el paciente y que puede comportar problemas en el ajuste farmacológico del tratamiento antipsicótico ${ }^{19}$. El $80 \%$ de los participantes que presentaron un periodo de abstinencia refirieron no haber sufrido un empeoramiento de la sintomatología psiquiátrica del trastorno de base, el $20 \%$ restante comenta mayores niveles de ansiedad (síntoma propio de la abstinencia a la nicotina). Otra creencia encontrada es que las prohibiciones totales de fumar aumenten el estrés y la ansiedad en el paciente, provocando un mayor número de alteraciones de conducta y solicitud de altas voluntarias ${ }^{20}$. Sin embargo, son numerosos los estudios que avalan la aceptación y la eficacia de la prohibición total de fumar en las unidades de hospitalización de salud mental, sin haber aumentado las alteraciones conductuales ni el estrés de los pacientes ${ }^{20,21}$. En este estudio se muestra cómo los niveles de craving de los participantes eran menores en los servicios de hospitalización, donde existe una prohibición total de fumar en ellos, objetivándose mayores tasas de craving entre la población de atención comunitaria y una puntuación de sintomatología abstinencial similar en los tres servicios. Se puede deducir de este hecho que la prohibición completa de fumar durante la hospitalización sirve de ayuda para el control del consumo de tabaco. Según el estudio de Kulkarni M et al. ${ }^{22}$, el $48 \%$ de los profesionales de la salud mental consideran que el tratamiento del tabaquismo en sus pacientes no es su responsabilidad.

En este estudio, no se ha detectado el cese completo del consumo de tabaco entre los y las participantes, aunque sí que se objetivó un descenso importante del número de cigarrillos diarios. La reducción del consumo de tabaco como paso previo a la cesación completa es una de las estrategias recomendadas por los exper$\operatorname{tos}^{23}$. Es importante que se valide bioquímicamente con una reducción significativa de los niveles de $\mathrm{CO}$ en el aire espirado, tal y como se ha mostrado en este estudio, con una reducción de 0.6 puntos. El problema principal de la reducción del consumo de cigarrillos es que ninguna cantidad de cigarrillos fumados ha demostrado ser segura. Por eso se considera que hay una reducción real de daños si la disminución del consumo es un paso que facilite la cesación posterior.

Como limitación principal del estudio se encuentran las propias de los estudios cuasiexperimentales, como no disponer de un grupo control o la no aleatorización de la muestra, de ahí que se haya intentado suplir esta limitación con el abordaje en diferentes servicios de salud mental. Así mismo, aunque el tamaño muestral es reducido, es suficiente según los cálculos realizados.

\section{Financiación}

Para la realización de este estudio se ha contado con la Ayuda a Proyectos de Investigación por el Colegio Oficial de Enfermería de la Región de Murcia en la convocatoria para el año 2016 y una beca PERIS en la convocatoria 2016-2020.

Los autores declaran que no existe conflicto de intereses en la realización y publicación de este estudio.

\section{Conclusiones}

Las personas con un trastorno mental están motivadas e interesadas en dejar su consumo de tabaco, no objetivándose un empeoramiento de su trastorno de base durante el periodo de abstinencia. La prohibición completa de fumar durante la hospitalización supone una oportunidad de abordar esta temática y proporcionar tratamiento para conseguir la cesación. Las intervenciones destinadas a este fin han resultado ser eficaces. 


\section{Bibliografía}

1. Bushe $C$, Haddad P, Peveler R, Pendlebury J. The role of lifestyle interventions and weight management in schizophrenia. $J$ Psychopharmacol. 2005;19(6 Suppl):28-35.

2. Sobradiel N, García-Vicent V. Consumo de tabaco y patología psiquiátrica. Trastor Adict. 2007;9(1):31-8.

3. De Leon J, Diaz FJ. A meta-analysis of worldwide studies demonstrates an association between schizophrenia and tobacco smoking behaviors. Schizophr Res. 2005;76(2-3):135-57.

4. Marques de Oliveira R, Furegato ARF. The opinion of patients with mental disorder about tobacco and its prohibition in psychiatric hospitalization. Rev Esc Enferm USP. 2014;48(3):500-6.

5. Tsoi DT, Porwal M, Webster AC. Interventions for smoking cessation and reduction in individuals with schizophrenia. Cochrane database Syst Rev. 2013;(2):CD007253-CD007253.

6. Zvolensky MJ, Farris SG, Leventhal AM, Ditre JW, Schmidt NB. Emotional disorders and smoking: Relations to quit attempts and cessation strategies among treatment-seeking smokers. Addict Behav. 2015;40:126-31.

7. Beck AK, Baker AL, Todd J. Smoking in schizophrenia: Cognitive impact of nicotine and relationship to smoking motivators. Schizophr Res Cogn. 2015;2(1):26-32.

8. Eriksen M, Mackay J, Schluger NW, Islami F, Drope J, et al. The Tobacco Atlas. The Tobacco Atlas. 2015;47.

9. Minichino A, Bersani FS, Calò WK, Spagnoli F, Francesconi M, Vicinanza R, et al. Smoking behaviour and mental health disordersmutual influences and implications for therapy. Vol. 10, International Journal of Environmental Research and Public Health. 2013. p. 4790-811.

10. De Leon J, Diaz FJ. A meta-analysis of worldwide studies demonstrates an association between schizophrenia and tobacco smoking behaviors. Schizophr Res. 2005;76(2-3):135-57.

11. Veiga Rodeiro S. Influencia de la presencia de patología psiquiátrica en la cesación tabáquica Estudio de los pacientes tratados. Universidad de Cantabria; 2016.

12. Schroeder SA, Morris CD. Confronting a Neglected Epidemic: Tobacco Cessation for Persons with Mental Illnesses and Substance Abuse Problems. Annu Rev Public Health. 2010;31(1):297-314.

13. Rice VH, Stead LF. Nursing interventions for smoking cessation. Cochrane Database Syst Rev. 2008;(1):CD001188.

14. Salvador Llivina T, Córdoba García R, Planchuelo Santos MA, Nebot Adell M, Becoña Iglesias E, Barrueco Ferrero M et al. Guía para dejar de fumar. Ministerio de Sanidad, Servicios Sociales e Igualdad (MSC) y Comité Nacional para la Prevención del Tabaquismo (CNPT); 2005.

15. Carreras R, Castells B, Chuecos M, de la Rosa D, Feria I, Fernández E et al. Guía Recintes sense fum. Xarxa Catalana d’Hospitals sense fum. 2011.

16. Ballbè i Guibernau $M$, Bruguera i Cortada $E$, Cano Vega $M$, Delgado Almeda MT, Fernández Grané T, Fonseca Casals F et al. Guía de actuación de pacientes fumadores ingresados en unidades de Salud mental. Xarxa Catalana d'Hospitals sense fum. 2012.

17. Rockville MD. Treating Tobacco Use and Dependence:2008 Update. Tobacco Use and Dependence Guideline Panel. 2008.

18. Gilbody S, Peckham E, Man M-S, Mitchell N, Li J, Becque T, et al. Bespoke smoking cessation for people with severe mental ill health (SCIMITAR): a pilot randomised controlled trial. The Lancet Psychiatry. 2015;2(5):395-402.

19. Ratschen E, Britton J, McNeill A. Implementation of smoke-free policies in mental health in-patient settings in England. $\mathrm{Br} J$ Psychiatry. 2009;194(6):547-51.

20. Harris GT, Parle D, Gagn J. Effects of a tobacco ban on long-term psychiatric patients. J Behav Heal Serv Res. 2007;34(1):43-55.

21. El-Guebaly N, Cathcart J, Currie S, Brown D, Gloster S. Public Health and Therapeutic Aspects of Smoking Bans in Mental Health and Addiction Settings. Psychiatr Serv. 2002;53(12):1617-22.
22. Kulkami M, Huddlestone L, Taylor A, Sayal K, Ratschen E. A crosssectional survey of mental health clinicians' knowledge, attitudes, and practice relating to tobacco dependence among young people with mental disorders. BMC Health Serv Res. 2014;14:618.

23. Anthenelli R, Benowitz N, West R, Aubin L, McRae T, Lawrence D, et al. Neuropsychiatric safety and efficacy of varenicline, bupropion, and nicotine patch in smokers with and without psychiatric disorders (EAGLES): a double-blind, randomised, placebocontrolled clinical trial. Lancet (London, England). 2016;387:2507-20. 